\title{
Adenovirus-mediated GM-CSF gene and cytosine deaminase gene transfer followed by 5-fluorocytosine administration elicit more potent antitumor response in tumor-bearing mice
}

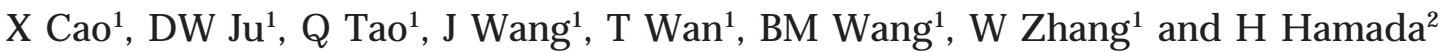 \\ ${ }^{1}$ Department of Immunology, Second Military Medical University, Shanghai, PR China; and ${ }^{2}$ Department of Molecular Biotherapy \\ Research, Japanese Foundation of Cancer Research, Tokyo, Japan
}

Antitumor effects of combined transfer of suicide and cytokine genes were investigated in this study. Adenovirus harboring $E$. coli cytosine deaminase gene (AdCD) and adenovirus harboring murine granulocyte-macrophage colony-stimulating factor gene (AdGMCSF) were used simultaneously for in vivo gene transfer in melanoma-bearing mice. Growth inhibition of established tumors and prolongation of survival period were observed more significantly in tumor-bearing mice after transfection with AdGMCSF and AdCD followed by continuous injection of prodrug 5-fluorocytosine (5FC) when compared with mice treated with control adenovirus AdlacZ/5FC, AdCD/5FC or AdGMCSF alone $(P<0.01)$. After combined therapy the expression of MHC-I $\left(H-2 D^{b}\right)$ and B7-1 molecules on freshly isolated tumor cells increased greatly and more dendritic cells and $C D 8^{+} T$ cells infiltrated into the tumor mass. The activity of specific cytotoxic $T$ lymphocytes was also found to be induced more significantly after the combined therapy. Further experiments showed that apoptosis of tumor cells and induction of antitumor immune response might be involved in the mechanisms of the tumor cell killing by the combined therapy. Our results demonstrated that combined transfer of the GM-CSF and CD suicide genes, being able to inhibit the growth of melanoma synergistically and induce specific antitumor immune response efficiently, thus addressing the drawbacks of suicide gene therapy or cytokine gene therapy which were proved to be not satisfactory when used alone, might be of therapeutic potential for gene therapy of cancer.

Keywords: cytosine deaminase; suicide gene; gene therapy; adenovirus; granulocyte-macrophage colony-stimulating factor; melanoma

\section{Introduction}

Gene therapy, as a promising treatment for many human modalities, encompasses efficient potentials for treating cancers that have failed conventional therapies. The use of suicide gene transfer for the therapy of cancer has been studied extensively. ${ }^{1-6}$ The principle underlying metabolic suicide gene therapy is the conversion of a relatively nontoxic prodrug to its toxic metabolite by an enzyme expressed by the suicide gene, which is not normally present in the eukaryotic cells. Several enzyme/prodrug systems, such as herpes simplex thymidine kinase (HSVtk), E. coli cytosine deaminase (CD) and varicella zoster thymidine kinase, have been developed for the treatment of tumors both in animal models and in patients. ${ }^{7-11} \mathrm{CD}$ enzyme exists in many bacteria and fungi but not in mammalian cells. 5-Fluorocytosine (5FC), being widely used for the treatment of fungal and bacterial infections, could be metabolized by CD to 5 -fluorouracil (5-FU), a highly toxic agent for the therapy of

Correspondence: X Cao, Department of Immunology, Second Military Medical University, 800 Xiang Yin Road, Shanghai 200433, PR China Received 17 November 1997; accepted 24 April 1998 various kinds of tumors. The growth of tumor cells modified to express CD could be significantly inhibited by $5 F C$ both in vitro and in vivo. Virus-directed enzyme/prodrug therapy (VDEPT), an approach exploiting the transcription differences between normal and neoplastic cells, has been used for the selective expression of CD in colorectal carcinoma cells. ${ }^{12,13}$ Therapy with suicide gene transfection was sometimes effective, but at other times it was reported to be not efficient in the induction of antitumor immunity, and tumor recurrence or metastasis often occurred after therapy. $2,14,15$

Direct transfer of cytokine genes into the tumor-bearing host has been demonstrated to be effective for the inhibition of tumor growth. We have shown that intratumoral injection of recombinant vaccinia virus encoding granulocyte-macrophage colony-stimulating factor (GMCSF) could exhibit significant antitumor response in melanoma-bearing mice. ${ }^{16}$ Tumor cells transduced with cytokine genes showed increased immunogenicity and decreased tumorigenicity. Tumor vaccine prepared with cytokine gene-modified tumor cells not only induced cellular immunity to protect against the same cells but also elicited protective immunity to the parental tumor cells. ${ }^{17}$ The therapeutic efficacy of one cytokine gene transfection is often not satisfactory especially in pre- 
established tumors. We have reported that combined transfer of two cytokine genes, or transfer of one cytokine gene in combination with certain agents, such as interleukin 2 (IL-2) or chemotherapeutic drugs, could greatly increase the therapeutic efficacy and induce obvious antitumor immunity of the host. ${ }^{18-20}$ GM-CSF-secreting tumor cells were found to stimulate potent, specific and long-lasting antitumor immunity. ${ }^{21,22}$ It was reported that GM-CSF gene-modified tumor vaccine exhibited most efficient antitumor efficacy among various cytokine genemodified vaccines because the locally produced GM-CSF could stimulate the proliferation and differentiation of antigen-presenting cells, thus augmenting the antitumor response of the host.

In this report, we hypothesized that combined use of GM-CSF gene and CD suicide gene therapy might have more significant antitumor effects than GM-CSF gene therapy or CD suicide gene therapy which were proved to be not satisfactory when used alone. To test this hypothesis, we treated melanoma-bearing mice with adenovirus expressing murine GM-CSF in combination with adenovirus expressing E. coli $\mathrm{CD}$ followed by $5 \mathrm{FC}$ administration. Inhibition of tumor growth and prolongation of survival period were observed more obviously in tumor-bearing mice after combined therapy when compared with GM-CSF gene therapy or CD suicide gene therapy alone. The mechanisms of the antitumor response were also studied in this investigation.

\section{Results}

Inhibition of tumor growth and prolongation of survival period in tumor-bearing mice after combined therapy C57BL/ 6 mice were inoculated s.c. with $1 \times 10^{5}$ B16F10 melanoma cells and treated with PBS, AdlacZ/5FC, AdCD, AdCD/5FC, AdGMCSF or AdCD/5FC in combination with AdGMCSF (AdCD/5FC/AdGMCSF). The results in Figure 1a demonstrated that inhibition of melanoma growth could be observed most significantly in mice after the treatment with AdGMCSF in combination with AdCD followed by continuous injection of prodrug 5FC (AdCD/5FC/AdGMCSF) when compared with that in mice treated with control adenovirus Adlac $Z / 5 F C$, AdCD, AdCD/5FC, or AdGMCSF alone $(P<0.01)$. AdCD/5FC or AdGMCSF therapy alone also showed a marked inhibitory effect on tumor growth when compared with PBS control group $(P<0.05)$. Neither AdlacZ/5FC nor AdCD treatment without 5FC administration elicited antitumor effects in tumor-bearing mice.

Ten tumor-bearing mice in each group were observed for their survival period. The results in Figure $1 \mathrm{~b}$ demonstrated that the mice which received combined therapy of AdCD/5FC/AdGMCSF were able to survive much longer than all the other mice which received control therapies $(P<0.01)$. Five of 10 mice after AdCD/5FC/AdGMCSF combined therapy, one of 10 mice after AdGMCSF therapy and two of 10 mice after AdCD/5FC therapy were found to be tumor-free and lived more than 90 days, while the control animals after PBS or AdlacZ/5FC treatment died within 2 months.

\section{Induction of more potent specific antitumor immunity in} mice after combined therapy with AdCD/5FC/AdGMCSF Splenocytes from the dead tumor-bearing mice after various therapies were used in cytolytic assays against YAC-

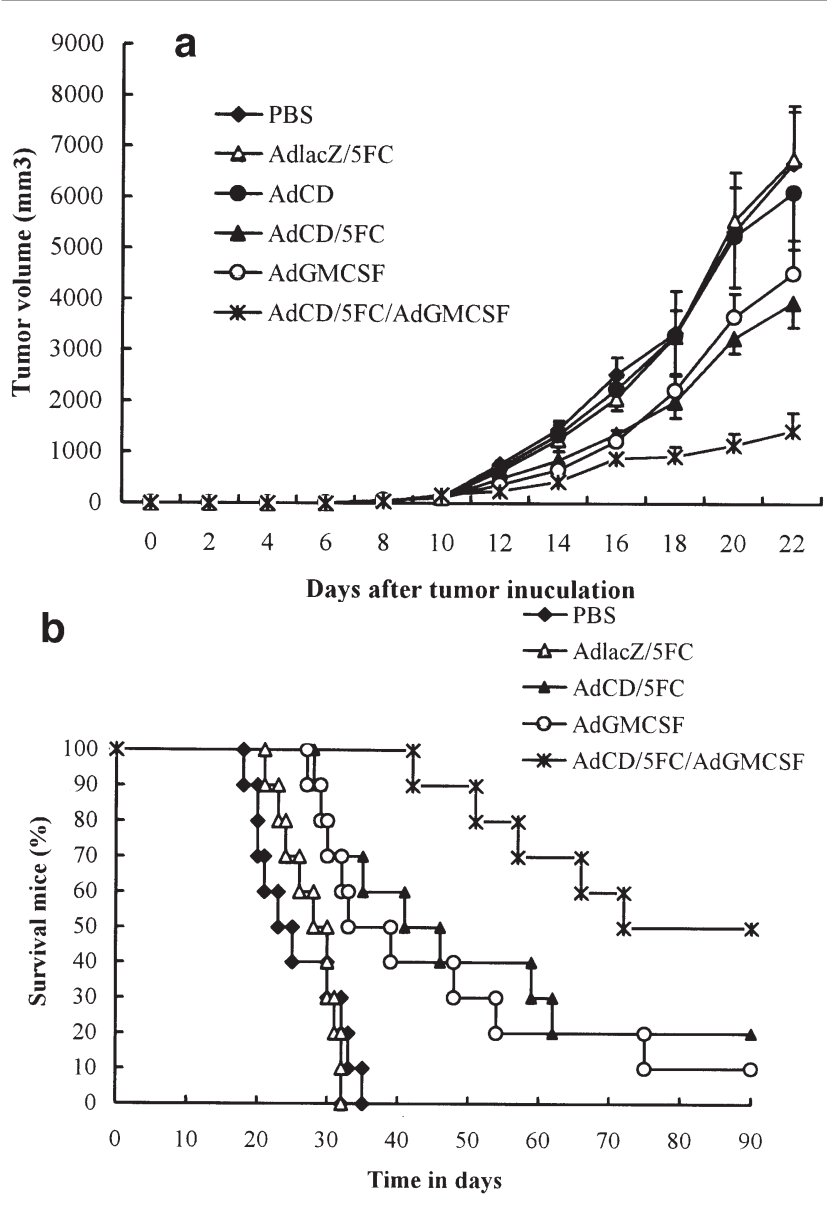

Figure 1 (a) Growth suppression of established B16F10-derived subcutaneous tumors by combined therapy of AdCD/5FC and AdGMCSF. The mice were inoculated with B16F10 melanoma cells on day 0, and then injected with recombinant adenovirus on day 3 and day 10, and received intraperitoneal injection of 5FC $300 \mathrm{mg} / \mathrm{kg}$ daily from day 4 to day 13 . Tumor volume was expressed as $0.5 \times$ length $\times$ width ${ }^{2}$. (b) Survival period of tumor-bearing mice after injection of AdCD/5FC and/or AdGMCSF.

1 cells or B16F10 cells at effector:target (E:T) ratios of 10:1, 20:1 and $40: 1$ by a $4-h{ }^{51} \mathrm{Cr}$ release assay. No increased NK activity was observed in mice after combined treatment when compared with that in mice in other groups, suggesting that the antitumor effect of AdCD/5FC/AdGMCSF was not mediated through the augmentation of NK activity (Figure 2a). Activity of cytotoxic T lymphocytes in spleen was tested utilizing B16F10 tumor cells as targets. The data in Figure $2 b$ demonstrated that the lymphocytes from AdCD/5FC and AdGMCSF treated mice showed an obvious increase of cytotoxic activity against B16F10 cells when compared with these lymphocytes from PBS, AdlacZ/5FC, AdCD $/ 5 F C$ or AdGMCSF-treated mice $(P<0.01)$. The cytolytic activity of lymphocytes in AdGMCSF-treated mice against B16F10 cells was also found to be higher than that in PBS, AdlacZ/5FC, or AdCD/5FC-treated mice $(P<0.05)$. No cytotoxicity effect was found with these lymphocytes when syngeneic erythroleukemia FBL3 cells were used as targets. These data suggested that the augmentation of tumor-specific immunity, not nonspecific immunity, might be involved in the antitumor response of the combined therapy. 

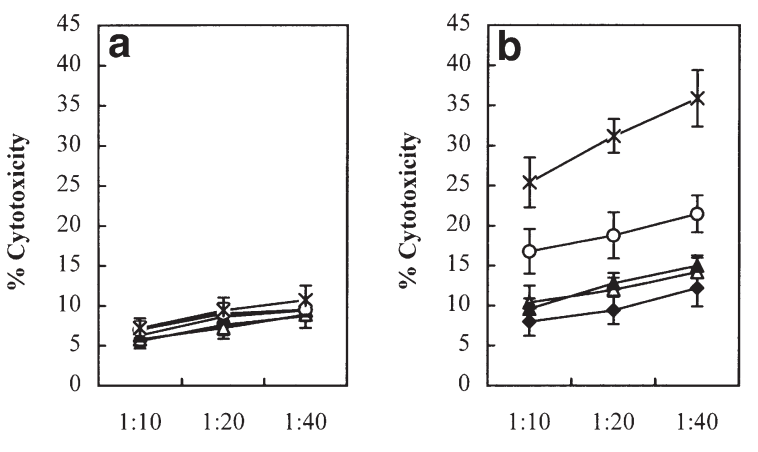

E:T E:T

* $\mathrm{AdCD} / 5 \mathrm{FC} / \mathrm{AdGMCSF}$

Figure 2 Cytotoxicity of NK (a) and CTL (b) derived from splenocytes of the tumor-bearing mice after treatment with AdCD/5FC and/or AdGMCSF. Cytotoxicity was determined by a standard $4-h{ }^{51} \mathrm{Cr}$ release assay by utilizing YAC-1 cells and B16F10 cells as targets, respectively.

\section{Augmentation of immunity in micro-environment of the tumor after combined therapy}

MHC class I and costimulatory molecule B7-1 molecule expression on the tumor cells after combined therapy with CD suicide gene transfer and GM-CSF gene transfer were determined by flow cytometry. As shown in Figure 3, B16F10 cells freshly isolated from AdlacZ/5FCor AdCD/5FC-treated mice expressed similar levels of MHC-I and B7-1 when analyzed with PE-conjugated monoclonal antibodies. Treatment of the mice with AdGMCSF in the presence or absence of AdCD/5FC, could augment the expression of MHC-I and B7-1 molecules on the tumor cells significantly when compared with treatment of the mice with AdlacZ/5FC or $\mathrm{AdCD} / 5 \mathrm{FC}$. In vitro infection of $\mathrm{B} 16 \mathrm{~F} 10$ cells with AdCD/AdGMCSF showed similar up-regulation of B71 and MHC-I molecules on these melanoma cells (data not shown). As shown in Figure 3 , more $\mathrm{CD}^{+} \mathrm{T}$ cell infiltration in tumors was observed in mice after combined therapy than that in mice after treatment with AdlacZ/5FC, AdCD/5FC or AdGMCSF. To determine if dendritic cells infiltrated into the tumor mass after various treatments, monoclonal antibody against NLDC145, a specific marker of dendritic cells, was used to label the dendritic cells in tumor cell suspension. The results in Figure 3 showed that the tumors in mice after administration with AdGMCSF or AdCD/5FC/AdGMCSF were infiltrated with dendritic cells, while few dendritic cells were found in AdlacZ/5FC- or AdCD/5FC-treated mice. These data suggested that augmentation of immunity in the micro-environment of the tumor could be achieved through the induction of $\mathrm{CD}^{+} \mathrm{T}$ cells and infiltration of dendritic cells after the combined therapy with AdCD/5FC and AdGMCSF.

\section{Mechanisms involved in the antitumor effects of suicide gene therapy}

First, we used flow cytometry for the determination of the apoptosis of tumor cells both in vitro and in vivo after treatment with AdlacZ/5FC, AdCD/5FC, AdGMCSF or

AdCD/5FC/AdGMCSF. The results in Figure 4 illustrated that neither treatment of the B16F10 melanoma cells with AdlacZ/5FC nor with AdGMCSF induced apoptosis both in vitro and in vivo. Obvious apoptosis was found with the B16F10 cells after treatment with AdCD/5FC, with or without AdGMCSF injection.

Second, in order to confirm further the assumption that antitumor immunity of the tumor-bearing mice was induced after the combined therapy, we have carried out another experiment using ex vivo AdCD/AdGMCSF transfected cells and in vivo tumor challenge. B16F10 cells were transfected with AdCD/AdGMCSF at MOI of 20:1, and $10^{5}$ virus-transfected tumor cells were inoculated s.c. into C57BL/6 mouse. Twenty-four hours after tumor inoculation 5FC $300 \mathrm{mg} / \mathrm{kg}$ was administered s.c. for 7 consecutive days. One week after the final injection of $5 F C$, PBS, $5 \times 10^{4}, 10^{5}$, or $2 \times 10^{5}$ wild-type B16F10 cells were inoculated s.c. into the immunized mice or control normal mice. All the control mice developed tumors and $90 \%$ of the mice died within 4 weeks after tumor inoculation. The mice inoculated with virus-treated tumor cells and injected with 5FC did not develop tumors within 4 weeks. The immunized mice showed significant protection against additional challenge with $5 \times 10^{4}, 10^{5}$, or $2 \times 10^{5}$ wild-type B16F10 cells. No palpable tumors were found in mice in these three groups 4 weeks after tumor challenge. These data suggested that AdCD/AdGMCSF transfection followed by 5FC administration generated potent antitumor immunity of the host.

\section{Discussion}

Suicide gene therapy is now widely investigated for the therapy of malignant tumors, selective killing of parasites, control of HIV infection, allogeneic bone marrow transplantation, and vascular smooth muscle cell proliferation, in which suicide gene expression in target cells renders them susceptible to killing by a non-toxic prodrug.,23-26 A recent study has demonstrated that glioma cells transduced with a CD/HSVtk fusion gene could exhibit enhanced metabolic suicide and radiosensitivity. ${ }^{27}$ Tissue-specific promoters such as alpha-fetoprotein (AFP), carcinoembryonic antigen (CEA) or ERBB2 transcriptional regulators were used for selected delivery of suicide genes into tumors. ${ }^{28-30}$ A phase I-II clinical study of the treatment of metastatic malignant melanoma using suicide gene therapy suggested that the localized tumoricidal activity may trigger a more general antineoplastic action, by facilitating a specific antitumoral immune response. A therapeutic trial on recurrent brain tumors or metastases has been conducted in the USA using a similar approach. ${ }^{31}$ The mechanisms of antitumor response by suicide gene therapy were found to be related to the induction of antitumor immunity and transfer of toxic drug metabolites. . $^{32,33}$

When cytokine genes were transferred for the therapy of established cancer, the antitumor immunity of the host could be augmented but it was usually too weak to eradicate established tumors. When CD suicide gene was transfected in vivo followed by the administration of prodrugs, the pre-established tumors could be easily eradicated, but it lacked the ability to induce an efficient antitumor immune response in the host. In this study adenovirus harboring CD gene or murine GM-CSF gene were used simultaneously for gene transfer in melanoma- 
MHC-1
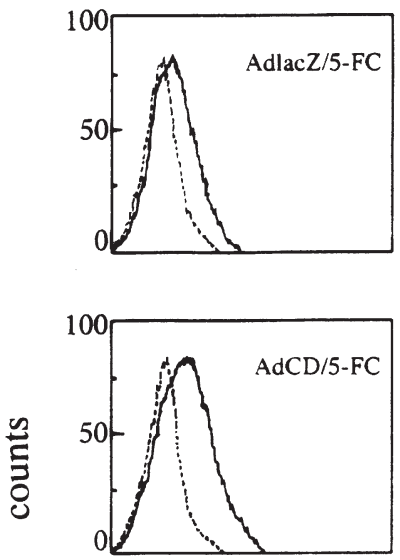

$\overline{0}$
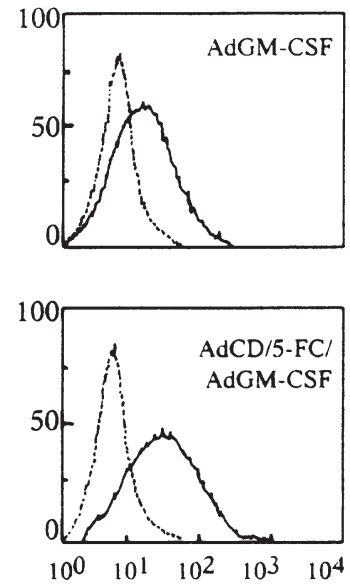

B7-1
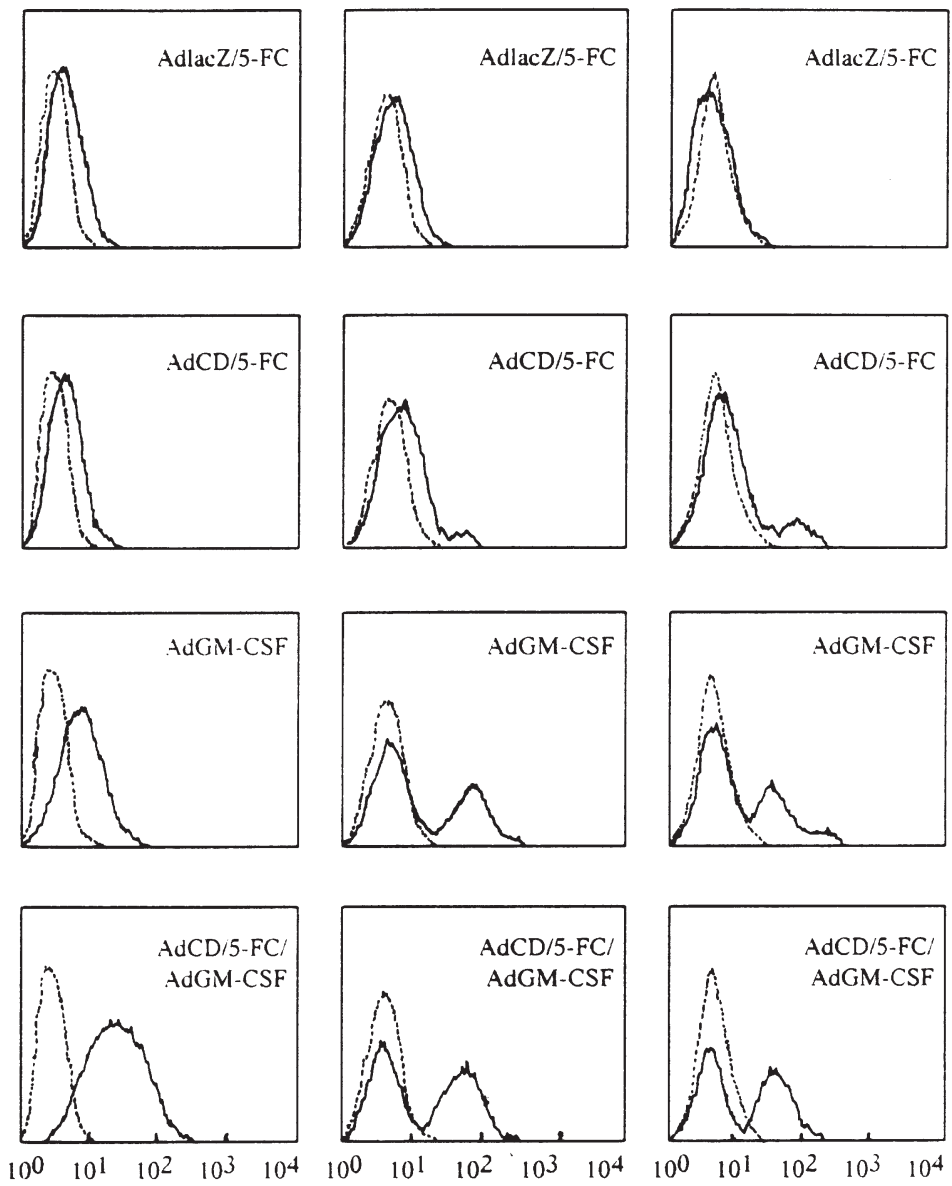

\section{Fluorescence}

Figure 3 FACS analysis of MHC-I, B7-1 molecule expression on freshly isolated tumor cells, and CD8 $8^{+}$cells, dendritic cells infiltrated in tumor mass of tumor-bearing mice after adenovirus-mediated CD suicide gene and/or murine GM-CSF gene therapy followed by 5FC administration. Monoclonal antibodies against MHC-I $\left(H-2 D^{b}\right), B 7-1$, dendritic cells (NLDC145) and CD8 $8^{+}$cells were used for FACS analysis. A Becton Dickinson FACScalibur flow cytometer was used for flow cytometry.

bearing mice. Significant inhibition of pre-established tumor growth and prolongation of survival period were observed in mice after the combined therapy with AdCD/5FC/AdGMCSF. Chen et $a l^{34,35}$ have investigated the effectiveness of combination therapy using a HSVtk suicide gene and an IL-2 gene for the treatment of metastatic colon carcinoma in the mouse liver. When poorly immunogenic tumor cells were genetically transduced to simultaneously express the cytokine IL-6 and CD, the tumor cells (205-IL6-CD) became highly immunogenic and could be rejected by normal mice without 5FC treatment. Mice with pre-existing wild-type pulmonary micrometastases exhibited prolonged survival and an increased rate of cure when treated with live 205-IL6-CD cells as a therapeutic vaccine. ${ }^{36}$ When tumor cells expressing IFN $\alpha$ gene and HSVtk gene were used as vaccine for the treatment of Friend erythroleukemia tumors, complete tumor rejection and cure was observed in more than $70 \%$ of the animals. ${ }^{37}$ These data demonstrated that the combination of cytokine and suicide gene therapy could be more efficient in the induction of antitumor immunity of the host.

Dendritic cells are professional antigen-presenting cells that play a major role in $\mathrm{T}$ cell-mediated immune responses. Recent studies have demonstrated that dendritic cells actively participated in the process of specific tumor antigen presentation. Several studies have documented that $\mathrm{CD}^{+} \mathrm{T}$ cells stimulated by dendritic cells could elicit potent antitumor response. ${ }^{38,39}$ Adenovirusmediated GM-CSF gene transfer in vivo was reported to induce dendritic cells to infiltrate into tumor mass. ${ }^{39} \mathrm{~A}$ large number of dendritic cells were also reported at the site of inoculation with GM-CSF-transfected melanoma cells. ${ }^{21,40}$ In this study we found obvious infiltration of dendritic cells in tumor mass after AdCD/5FC/AdGMCSF therapy. The mechanisms involved in the augmentation of tumor-specific immunity by locally produced GM-CSF is still not clear. The dendritic cells may be derived from the differentiation of hematopoietic precursors induced by GM-CSF ${ }^{41}$ and the 


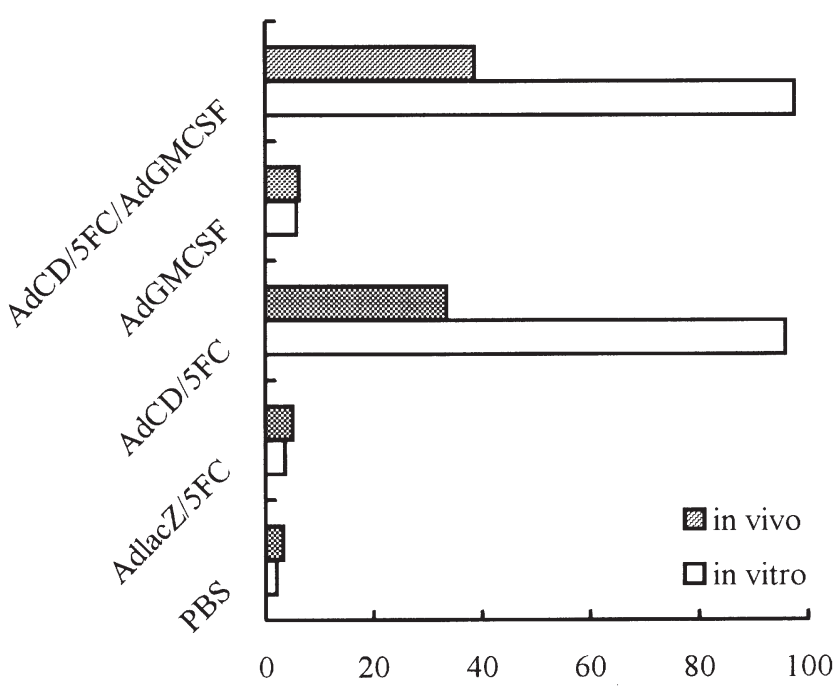

Apoptosis (\%)

Figure 4 Apoptosis analysis of B16F10 melanoma cells after treatment with AdCD/5FC and/or AdGMCSF. B16F10 cells from continuous culture in vitro or freshly isolated from dead tumor-bearing mice were stained and apoptosis determined on a Becton Dickinson FACScalibur flow cytometer.

presence of GM-CSF at the site of the tumor may attract dendritic cells from surrounding tissues. ${ }^{39}$ These dendritic cells may play important roles in the processing and presentation of tumor antigens released from AdCD/5FC killed tumor cells, thus enhancing the induction of specific antitumor immune response.

Expression of MHC class I molecule $\left(\mathrm{H}-2 \mathrm{D}^{\mathrm{b}}\right.$ in C57BL/6 mouse) and B7-1, a well identified costimulatory molecule, is very important for the presentation of tumor antigen and the activation of $\mathrm{CD}^{+} \mathrm{T}$ cells. Ramesh et $a l^{42}$ have found that expression of costimulatory molecules B7 and ICAM could be up-regulated after transfection with the HSVtk suicide gene. In the present study, we have demonstrated that expression of $\mathrm{H}-2 \mathrm{D}^{\mathrm{b}}$ and $\mathrm{B} 7-$ 1 on freshly isolated tumor cells increased markedly after CD suicide gene therapy in combination with GM-CSF gene therapy. We speculated that the tumor cells, with increased MHC-I and costimulatory molecule B7 expression, may serve as the source of tumor-specific antigen, as well as antigen-presenting cells. In order to investigate if this altered micro-environment of tumor affected the antitumor properties of host lymphocytes, we studied the effector functions of lymphocytes both in the spleen and the tumor mass. Consistent with the above results, we have also demonstrated that more $\mathrm{CD} 8^{+} \mathrm{T}$ cells infiltrated into and around the tumor mass after combined gene therapy. Cytotoxicity analysis showed that NK activity of the splenocytes did not change markedly after combined therapy, but the activity of specific cytotoxic $\mathrm{T}$ lymphocytes from the spleen in tumor-bearing mice treated with $\mathrm{AdCD} / 5 \mathrm{FC}$ in combination with AdGMCSF gene transfection increased significantly. The increase in the CTL activity suggested that the antitumor effect of the combined therapy might be through the induction of specific antitumor immunity, not non-specific NK activity, of the melanoma-bearing mice.

In vivo transfer of CD gene followed by 5FC adminis- tration is currently being frequently evaluated in animal models for the gene therapy of cancer. Its therapeutic effect is based on a 'bystander effect' whereby CD genemodified tumor cells are toxic to nearby unmodified tumor cells when exposed to the antiviral drug 5FC. Induction of antitumor immune response of the host is obviously very important for its mechanisms of tumor cell killing. We also found obvious apoptosis of tumor cells both in vitro and in vivo induced by AdCD/5FC treatment, and the released antigen from the dead tumor cells might be harvested and processed by the GM-CSF activated antigen-presenting cells. This is different from ganciclovir, which is metabolized by HSVtk and elicits 'bystander effects' via the 'gap junction' of the tumor cells. ${ }^{43}$ Immunization with CD/GM-CSF gene transfected B16F10 melanoma cells followed by 5FC administration generated significant protection against challenge with wild-type B16F10 melanoma cells. Therefore, these findings might help to understand the mechanism of more significant tumor cell killing in vivo using CD gene transfection followed by 5FC administration in combination with GM-CSF gene transfection.

In conclusion, we have shown that combined transfer of $E$. coli CD suicide gene and GM-CSF gene followed by continuous injection of prodrug $5 \mathrm{FC}$ could inhibit the growth of pre-established melanoma synergistically, partially through the efficient induction of specific antitumor immune response. We speculated that the tumor cell debris formed after AdCD/5FC treatments was processed and presented to the immune system of the host by antigen-presenting cells, for example, dendritic cells induced by GM-CSF gene transfection. In addition, the tumor cells, which expressed more MHC-I and B7 molecules after AdCD/5FC/AdGMCSF combined therapy, might activate antitumor immune response by acting as antigen-presenting cells themselves. The combined therapy, being able to address the drawbacks of cytokine gene therapy or suicide gene therapy which were proved to be not satisfactory when used alone, might be of great interest for gene therapy of cancer.

\section{Materials and methods}

\section{Animals and cell lines}

Male or female C57BL/6 mice, 6-8 weeks of age, purchased from Joint Ventures Sipper BK Experimental Animals, Shanghai, China, were housed for 1 week in a specific pathogen-free state for experiment. B16F10, a subclone of B16 melanoma cell line from C57BL/6 and 293, a continuous cell line derived from human embryonic kidney were maintained in RPMI-1640 medium supplemented with penicillin $100 \mathrm{U} / \mathrm{ml}$, streptomycin 100 $\mu \mathrm{g} / \mathrm{ml}, 2$-mercaptoethanol $50 \mathrm{mmol} / 1$ and $10 \%$ fetal calf serum (FCS). All culture media were purchased from Gibco-BRL (Gaithersburg, MD, USA) and FCS provided by Shanghai Institute of Biological Products, Shanghai, China.

\section{Preparation of recombinant adenoviruses}

Three replication-defective recombinant adenoviruses, AdlacZ (harboring the $\beta$-galactosidase gene), AdCD (harboring the E. coli CD gene), and AdGMCSF (harboring the murine GM-CSF gene), were constructed from human adenovirus serotype 5 using homologous 
recombination. ${ }^{21,44}$ The expression of these genes was driven by a CAG promoter. The recombinant adenoviruses were propagated with 293 cells and the titers of the adenoviral preparations were determined by plaqueforming assay on 293 cells. Briefly, serial 10-fold dilutions of adenovirus were added to 24-well plates (Corning, NY, USA), containing confluent 293 cell monolayers. After 48 $\mathrm{h}$ of incubation in a humidified atmosphere, the end point of $50 \%$ infectivity was determined according to the cytopathic effect. The recombinant adenoviruses produced were diluted to a titer of $10^{10}$ p.f.u. $/ \mathrm{ml}$ in PBS and stored at $-70^{\circ} \mathrm{C}$.

\section{Experimental groups and therapeutic regimens}

B16F10 melanoma cells were taken from continuous culture and resuspended in PBS without FCS for inoculation into mice. C57BL/ 6 mice were inoculated s.c. with $1 \times 10^{5}$ B16F10 cells. Three days after tumor inoculation, the tumor-bearing mice were divided into six groups with 15 mice in each group and the mice in each group were injected intratumorally with any of the following preparations: PBS, AdlacZ, AdCD (without 5FC administration), AdCD, AdGMCSF, AdCD plus AdGMCSF. Injections of the viruses of $10^{9}$ in $0.1 \mathrm{ml}$ PBS were performed 3 days after the inoculation of tumor cells and a booster of the same injection was given after 7 days. Twenty-four hours after the first injection of adenoviruses 5FC (Sigma, St Louis, MO, USA) 300 $\mathrm{mg} / \mathrm{kg}$ was injected daily i.p. into AdlacZ-, AdCD-, or AdCD/AdGMCSF-treated mice for 10 consecutive days. The length and width of the tumor mass were measured with a caliper every other day after tumor inoculation and the tumor volume were expressed as $0.5 \times$ length $\times$ width $^{2}$.

\section{Cytotoxic assay}

Splenic lymphocytes were isolated from dead tumorbearing mice 3 days after the last injection of 5FC and directly used as NK effector cells. The lymphocytes were cocultured with inactivated B16F10 cells for 7 days, and then collected as CTL effector cells. The NK activity and CTL activity were determined by a standard $4-h{ }^{51} \mathrm{Cr}$ release assay. $2 \times 10^{6}$ YAC-1 or B16F10 cells in $0.5 \mathrm{ml}$ RPMI-1640 with 20\% FCS were labeled with $200 \mu \mathrm{Ci}$ $\mathrm{Na}^{51} \mathrm{CrO}_{4}$ (Amersham, Arlington Heights, IL, USA) for $2 \mathrm{~h}$. The labeled cells were washed three times in serumfree medium. $10^{4}$ Target cells were then mixed with effector cells for $4 \mathrm{~h}$ at $37^{\circ} \mathrm{C}$ at the ratio indicated. For the maximal ${ }^{51} \mathrm{Cr}$ release control, $0.1 \mathrm{ml}$ of $0.1 \mathrm{~N} \mathrm{HCl}$ was added to the target cells, and for the spontaneous ${ }^{51} \mathrm{Cr}$ control, $0.1 \mathrm{ml}$ medium was added to the labeled cells. The amount of ${ }^{51} \mathrm{Cr}$ released was determined by $\gamma$ counting on a 1275 Minigamma Counter (LKB-Wallac, Finland) and the percentage of specific lysis was calculated as follows:

$$
\begin{aligned}
& \% \text { of specific lysis }= \\
& \frac{\text { Experimental c.p.m. }- \text { spontaneous c.p.m. }}{\text { Maximal c.p.m. }- \text { spontaneous c.p.m. }} \times 100 \text {. }
\end{aligned}
$$

\section{Flow cytometry analysis}

A tumor nodule was taken from the dead mice 3 days after the administration of 5FC and the tumor mass was minced. The tumor cells or infiltrated lymphocytes were isolated from the cell suspensions utilizing Ficoll-
Hypaque solution. The isolated tumor cells were confirmed to be free of infiltrated immune cells by means of histochemistry. The tumor cells were incubated with optimal concentrations of PE-conjugated monoclonal antibodies against MHC-I (H-2D $\left.{ }^{\mathrm{b}}\right)$ or B7-1 (Pharmingen, San Diego, CA, USA) for $30 \mathrm{~min}$ at $4^{\circ} \mathrm{C}$. The tumor cells were washed three times with cold PBS and resuspended in PBS containing 1\% formaldehyde. The lymphocytes were incubated with rat anti-mouse CD8 monoclonal antibodies (Pharmingen) for $30 \mathrm{~min}$ followed by washing with cold PBS three times. The lymphocytes were then incubated with FITC-conjugated goat anti-rat IgG. The lymphocytes were washed with PBS and resuspended in PBS containing 1\% formaldehyde. The tumor cell suspension without separation was directly used for dendritic cell analysis with rat anti-mouse monoclonal antibody NLDC145 (gifts from Dr Z Qin, MDC, Germany) as mentioned for $\mathrm{CD}^{+} \mathrm{T}$ cell analysis. Control incubations used for determining levels of background staining with the second antibody were irrelevant normal rat anti-mouse IgG. Flow cytometry was analyzed on a FACScalibur flow cytometer (Becton Dickinson, San Jose, CA, USA).

\section{Apoptosis assay of B16F10 cells}

To determine if apoptosis of tumor cells participated in the process of the bystander effect, we analyzed the percentages of tumor cells undergoing apoptosis. B16F10 cells from continuous cultivation in vitro were infected with adenoviruses at a MOI of 20:1 for $24 \mathrm{~h}$ followed by incubation with $3 \mathrm{~mm} 5 \mathrm{FC}$ for another $24 \mathrm{~h}$ at $37^{\circ} \mathrm{C}$, or B16F10 cells isolated from dead tumor-bearing mice were stained and apoptosis determined on a Becton Dickinson FACScalibur flow cytometer.

\section{Statistics}

All experiments were run in triplicate and the results are means \pm s.d. of triplicate determinations. Statistical analysis was performed using Student's $t$ test. A $P<0.05$ was considered to be statistically significant.

\section{Acknowledgements}

This work was supported by grants from the National Natural Science Foundation of China (No. 39600181) and National High Biotechnology Project of China (BH0301-03).

\section{References}

1 Deonarain MP, Spooner RA, Epenetos AA. Genetic delivery of enzymes for cancer therapy. Gene Therapy 1995; 2: 235-244.

2 Mullen CA. Metabolic suicide genes in gene therapy. Pharmac Ther 1994; 63: 199-207.

3 Tiberghien P. Use of suicide genes in gene therapy. J Leukocyte Biol 1994; 56: 203-209.

4 Kwong YL et al. Adenoviral mediated suicide gene therapy for hepatic metastases of breast cancer. Cancer Gene Ther 1996; 3: 339-344.

5 Izquierdo $\mathrm{M}$ et al. Human malignant brain tumor response to herpes simplex thymidine kinase (HSVtk)/ganciclovir gene therapy. Gene Therapy 1996; 3: 491-495.

6 Dilber MS et al. Suicide gene therapy for plasma cell tumors. Blood 1996; 88: 2192-2200.

7 Mullen CA, Coale MM, Lowe R, Blaese RM. Tumors expressing the cytosine deaminase suicide gene can be eliminated in vivo with 5-fluorocytosine and induce protective immunity to wildtype tumor. Cancer Res 1994; 54: 1503-1506. 
8 Austin EA, Huber BE. A first step in development of gene therapy for colorectal carcinoma: cloning, sequencing, and expression of Escherichia coli cytosine deaminase. Mol Pharmacol 1992; 43: 380-387.

9 Mullen CA, Kilstrup M, Blaese RM. Transfer of the bacterial gene for cytosine deaminase to mammalian cells confers lethal sensitivity to 5-fluorocytosine: a negative selection system. Proc Natl Acad Sci USA 1992; 89: 33-37.

10 Hirschowitz EA et al. In vivo adenovirus-mediated gene transfer of the Escherichia coli cytosine deaminase gene to human colon carcinoma-derived tumors induces chemosensitivity to 5-fluorocytosine. Hum Gene Ther 1995; 6: 1055-1063.

11 Szala S, Missol E, Sochanik A, Strozyk M. The use of cationic liposomes $\mathrm{C}$ CHOL/DOPE and DDAB/DOPE for direct transfer of Escherichia coli cytosine deaminase gene into growing melanoma tumors. Gene Therapy 1996; 3: 1026-1031.

12 Huber BE et al. In vivo antitumor activity of 5-fluorocytosine on human colorectal carcinoma cells genetically modified to express cytosine deaminase. Cancer Res 1993; 53: 4619-4626.

13 Huber BE et al. Metabolism of 5-fluorouracil in human colorectal tumor cells transduced with the cytosine deaminase gene: significant antitumor effects when only a small percentage of tumor cells express cytosine deaminase. Proc Natl Acad Sci USA 1994; 91: 8302-8306.

14 Barba D, Hardin J, Sadelain M, Gage FH. Development of antitumor immunity following thymidine kinase mediated killing of experimental brain tumors. Proc Natl Acad Sci USA 1994; 91: 4348-4351.

15 Consalvo $\mathrm{M}$ et al. 5-Fluorocytosine induced eradication of murine adenocarcinomas engineered to express the cytosine deaminase suicide gene requires host immune competence and leaves an efficient memory. J Immunol 1995; 154: 5302-5312.

$16 \mathrm{Ju}$ DW, Cao X, Acres B. Intratumoral injection of GM-CSF gene encoded recombinant vaccinia virus elicits potent antitumor response in a murine melanoma model. Cancer Gene Ther 1997; 4: 139-144.

17 Anderson WF. Gene therapy for cancer. Hum Gene Ther 1994; 5 $1-2$.

18 Cao X et al. Enhanced efficacy of combination of IL-2 gene and IL-6 gene transfected tumor cells in the treatment of established metastatic tumors. Gene Therapy 1996; 3: 421-426.

19 Cao X et al. Induction of antitumor immunity and treatment of preestablished tumors by inactivated IL- 6 gene transfected melanoma cells combined with low dose IL-2. J Cancer Res Clin Oncol 1995; 121: 721-728.

20 Cao $\mathrm{X}$ et al. Treatment of human hepatocellular carcinoma by fibroblast-mediated human interferon- $\alpha$ gene therapy in combination with adoptive chemoimmunotherapy. J Cancer Res Clin Oncol 1995; 121: 457-462.

21 Abe J et al. Antitumor effect induced by granulocyte/ macrophage colony-stimulating factor gene-modified tumor vaccination: comparison of adenovirus- and retrovirusmediated genetic transduction. J Cancer Res Clin Oncol 1995; 121 587-593.

22 Dranoff $G$ et al. Vaccination with irradiated tumor cells engineered to secrete murine granulocyte-macrophage colony-stimulating factor stimulates potent, specific and long lasting antitumor immunity. Proc Natl Acad Sci USA 1993; 90: 3539-3543.

23 Muyombwe A, Olivier M, Ouellette M, Papadopoulou B. Selective killing of Leishmania amastigotes expressing a thymidine kinase suicide gene. Exp Parasitol 1997; 85: 35-42.

24 Ohno T et al. Gene therapy for vascular smooth muscle cell proliferation after arterial injury. Science 1994; 265: 781-784.

25 Barinaga M. Gene therapy for clogged arteries passes test in pigs. Science 1994; 265: 738-741.
26 Rogers RP et al. Killing Epstein-Barr virus positive B lymphocytes by gene therapy: comparing the efficacy of cytosine deaminase and herpes simplex virus thymidine kinase. Hum Gene Ther 1996; 7: 2235-2245.

27 Rogulski KR, Kim JH, Kim SH, Freytag SO. Glioma cells transduced with an Escherichia coli CD/HSV1 TK fusion gene exhibit enhanced metabolic suicide and radiosensitivity. Hum Gene Ther 1997; 8: 73-85.

28 Richards CA, Austin EA, Huber BE. Transcriptional regulatory sequences of carcinoembryonic antigen: identification and use with cytosine deaminase for tumor specific gene therapy. Hum Gene Ther 1995; 6: 881-893.

29 Ring CJ, Harris JD, Hurst HC, Lemoine NR. Suicide gene expression induced in tumour cells transduced with recombinant adenoviral, retroviral and plasmid vectors containing the ERBB2 promoter. Gene Therapy 1996; 3: 1094-1103.

30 Harris JD et al. Gene therapy for cancer using tumor-specific prodrug activation. Gene Therapy 1994; 1: 170-175.

31 Klatzmann D. Gene therapy for metastatic malignant melanoma: evaluation of tolerance to intratumoral injection of cells producing recombinant retroviruses carrying the herpes simplex virus type 1 thymidine kinase gene, to be followed by ganciclovir administration. Hum Gene Ther 1996; 7: 255-267.

32 Freeman SM, Ramesh R, Marrogi AJ. Immune system in suicide gene therapy. Lancet 1997; 349: 2-3.

33 Ishii-Morita $\mathrm{H}$ et al. Mechanism of 'bystander effect' killing in the herpes simplex thymidine kinase gene therapy model of cancer treatment. Gene Therapy 1997; 4: 244-251.

34 Chen $\mathrm{SH}$ et al. Combination gene therapy for liver metastasis of colon carcinoma in vivo. Proc Natl Acad Sci USA 1995; 92 2577-2581.

35 Chen $\mathrm{SH}$ et al. Combination suicide and cytokine gene therapy for hepatic metastases of colon carcinoma: sustained antitumor immunity prolongs animal survival. Cancer Res 1996; 56: 37583762 .

36 Mullen CA, Petropoulos D, Lowe RM. Treatment of microscopic pulmonary metastases with recombinant autologous tumor vaccine expressing interleukin 6 and Escherichia coli cytosine deaminase suicide genes. Cancer Res 1996; 56: 1361-1366.

37 Santodonato $\mathrm{L}$ et al. Cure of mice with established metastatic friend leukemia cell tumors by a combined therapy with tumor cells expressing both interferon- $\alpha 1$ and herpes simplex thymidine kinase followed by ganciclovir. Hum Gene Ther 1996; 7: 1-10.

38 Gabilovich DI et al. Dendritic cells in antitumor immune response. II. Dendritic cells grown from bone marrow precursors but not mature DC from tumor-bearing mice are effective antigen carriers in the therapy of established tumors. Cell Immunol 1996; 170: 111-119.

39 Lee C-K et al. Genetic immunotherapy of established tumors with adenovirus-murine granulocyte-macrophage colony-stimulating factor. Hum Gene Ther 1997; 8: 187-193.

40 Armstrong CA et al. Antitumor effects of granulocyte-macrophage colony-stimulating factor production by melanoma cells. Cancer Res 1996; 56: 2191-2198.

41 Inaba K et al. Generation of large numbers of dendritic cells from mouse bone marrow cultures supplemented with granulocytemacrophage colony-stimulating factor. I Exp Med 1992; 176: 1693-1702.

42 Ramesh R et al. Expression of costimulatory molecules: B7 and ICAM up-regulation after treatment with a suicide gene. Cancer Gene Ther 1996; 3: 373-384.

43 Pitts JD. Cancer gene therapy: a bystander effect using the gap junction pathway. Mol Carcinogen 1994; 11: 127-130.

44 Kanai $\mathrm{F}$ et al. In vivo gene therapy for alpha fetoprotein producing hepatocellular carcinoma by adenovirus-mediated transfer of cytosine deaminase gene. Cancer Res 1997; 57: 461-465. 